\title{
PREVALENCE AND OUTCOME OF CLEAR CELL CARCINOMA AND ENDOMETRIOID CARCINOMA OF OVARY COEXISTING WITH ENDOMETRIOSIS
}

\author{
Jitima Tiyayon*, Raweewan Promsila** \\ ${ }^{*}$ Department of Obstetrics and Gynaecology, Rajavithi Hospital, College of Medicine Rangsit University \\ ${ }^{* *}$ Department of Obstetrics and Gynaecology, Sawanpracharak Hospital
}

There were studies proposed the association of endometriosis and ovarian cancer (1). In addition, the pathophysiology and clinical manifestation of endometriosis-associated ovarian cancer (EAOC) were widely reviewed (2). The risk of ovarian cancer from endometriosis ranged from 1.3 to 1.9 depending on histologic sub-type (1). Clear cell and endometrioid histology of ovarian cancer were reported to be closely associated with endometriosis with relative risk of 2.53 and 3.37 respectively (3-5).

The objective of the current article aimed to review the prevalence and outcome of endometriosis-associated ovarian cancer histologic-type clear cell carcinoma and endometrioid adenocarcinoma.

From 1 January 2005 to 31 December 2015, a total of 333 patients who had histologic subtype of clear cell carcinoma and endometrioid carcinoma were identified. 45 (13.51\%) had pathologically confirmed coexisting endometriosis, and 285 (85.58\%) had no endometriosis.

\begin{tabular}{|c|c|c|c|}
\hline Demographic data & $\begin{array}{l}\text { Endometriosis- } \\
\text { associated } \\
\quad n=45\end{array}$ & $\begin{array}{c}\text { Non } \\
\text { endometriosis- } \\
\text { associated } \\
\mathbf{n}=285 \\
\end{array}$ & $P$ value \\
\hline Age & 50.84 & 54.17 & 0.031 \\
\hline Symptoms & & & 0.376 \\
\hline - $\quad$ Abdominal mass & 2 & 6 & \\
\hline Abdominal pain & 29 & 209 & \\
\hline Abnormal uterine bleeding & 14 & 70 & \\
\hline Stage & & & 0.028 \\
\hline - I & $36(80)$ & $168(58.9)$ & \\
\hline II & $6(13.3)$ & $45(15.8)$ & \\
\hline III & $3(6.7)$ & $58(20.4)$ & \\
\hline IV & & $14(4.2)$ & \\
\hline Surgery & & & 0.002 \\
\hline - $\quad$ Optimal surgery & $35(77.8)$ & $156(54.7)$ & \\
\hline Suboptimal surgery & $10(22.2)$ & $76(26.7)$ & \\
\hline Incomplete staging & & $53(18.6)$ & \\
\hline Adjuvant chemotherapy & & & 0.720 \\
\hline Yes & $41(91.1)$ & $264(92.6)$ & \\
\hline No & $4(8.9)$ & $21(7.4)$ & \\
\hline Intra-operative complications & & & 0.054 \\
\hline - Yes & $0(0)$ & $22(7.7)$ & \\
\hline No & $45(100)$ & $263(92.3)$ & \\
\hline Post-operative complications & & & 0.002 \\
\hline - No & $35(77.8)$ & $156(54.7)$ & \\
\hline Wound dehiscence & $10(22.2)$ & $76(26.7)$ & \\
\hline Septicemia & $0(0)$ & $53(18.6)$ & \\
\hline
\end{tabular}

\begin{tabular}{|c|c|c|c|}
\hline $\begin{array}{c}\text { Comparing clear cell } \\
\text { carcinoma and } \\
\text { endometrioid carcinoma }\end{array}$ & $\begin{array}{c}\text { Clear cell } \\
\text { carcinoma } \\
\mathbf{n}=31\end{array}$ & $\begin{array}{l}\text { Endometrioi } \\
\text { d carcinoma } \\
n=14\end{array}$ & $P$ value \\
\hline Age & 50.77 & 51 & 0.937 \\
\hline Symptoms & & & 0.002 \\
\hline Abdominal mass & 25 & 4 & \\
\hline Abdominal pain & 0 & 2 & \\
\hline Abnormal uterine bleeding & 6 & 8 & \\
\hline Stage & & & 0.714 \\
\hline I & $24(77.4)$ & $12(85.7)$ & \\
\hline II & $5(16.1)$ & $1(7.1)$ & \\
\hline III & $2(6.5)$ & $1(7.1)$ & \\
\hline IV & $0(0)$ & $0(0)$ & \\
\hline Surgery & & & 0.491 \\
\hline Optimal surgery & $25(80.6)$ & $10(71.4)$ & \\
\hline Suboptimal surgery & $6(19.4)$ & $4(28.6)$ & \\
\hline Incomplete staging & $0(0)$ & $0(0)$ & \\
\hline Adjuvant chemotherapy & & & 0.782 \\
\hline Yes & $28(90.3)$ & $13(92.9)$ & \\
\hline No & $3(9.7)$ & $1(7.1)$ & \\
\hline
\end{tabular}

There was significant higher probability of optimal surgery in EAOC compared to non-EAOC $(77.8 \%$ and $54.7 \%$ respectively) as well as post-operative complication which was revealed lower incidence of EAOC $(22.2 \%, \mathrm{p}=0.02)$.

\section{Intraoperative complication}

\begin{tabular}{|l|c|c|}
\hline \multicolumn{1}{|c|}{ Type of complications } & No. & $\%$ \\
\hline Blood loss, anemia & 11 & 50 \\
\hline Bladder and ureteric injury & 6 & 27.3 \\
\hline Bowel injury & 5 & 22.7 \\
\hline
\end{tabular}

Median follow-up time in this study was 27.95 months (range from 0 to 134 months), 38 of 45 (84\%) patients with EAOC demonstrated normal clinical symptoms at the time of last follow-up, while 1 (2\%) had progression of disease, 5 (11\%) had loss to follow-up, and 1 (2\%) had acute renal failure. In non-EAOC, 201 of 284 (70\%) patients had normal clinical symptoms at last follow-up date, followed by 42 (14\%) patients had disease progression, 11 (3.8\%) patients had stable disease, and 23 (8\%) had loss to follow-up. There was significant probability of treatment outcome in EAOC $(p=0.023)$.

1.Gynecologic oncology. 2006;101(2):331-41

2. Int J Gynecol Pathol. 2011;30(6):553-568.

3. Int J Mol Sci. 2013;14:5367-5379.

4. Japan. Int J Gynecol Cancer. 2007;17: 37-43. 